\title{
Seguridad de la corrección percutánea de cardiopatías congénitas en mujeres en sus primeros días posparto
}

\author{
Safety of percutaneous correction of congenital \\ heart disease in women in their early postpartum \\ days
}

\author{
Juan Manuel Senior, Andrés Fernández, Arturo Rodríguez, \\ Diana P. CÁrdenas, Erick Trespalacios, Roberto Trespalacios, \\ Jorge M. Gomez • Medellín (Colombia)
}

\section{Resumen}

Introducción: las cardiopatías congénitas en el embarazo son causa importante de morbimortalidad materna, con todas las consecuencias sociales y económicas que esto conlleva. Hasta el momento las correcciones de las cardiopatías detectadas durante el embarazo se realizan en el periodo posterior a los 6 meses después del parto, teniendo en cuenta la reversión de los cambios fisiológicos propios de este y su falta de tratamiento aumenta la morbimortalidad. En nuestro medio las correcciones diferidas no llegan a realizarse por las dificultades en el sistema de salud y las condiciones socioculturales de las pacientes, que implican perdida del seguimiento, lo que impide la corrección oportuna de los defectos y desarrollo de síndrome de Eisenmenger.

Objetivo: evaluar la seguridad de la corrección percutánea de cardiopatías en mujeres en los primeros días después de su parto y los resultados de la misma a corto y largo plazo.

Metodología: estudio descriptivo, serie de casos.

Resultados: se reportan ocho casos de cierre por vía percutánea con éxito en: cuatro pacientes con comunicación interauricular y cuatro con ductus arterioso persistente en los primeros días después del parto (promedio de 8.7 días) con buenos resultados clínicos para el producto (APGAR promedio al minuto de 6.8 y de 8.7 a los cinco minutos), y para la madre, con alta hospitalaria temprana ( $87.5 \%$ en las primeras 24 horas después del procedimiento), con $62.5 \%$ de las madres asintomáticas a los nueve meses después de haber sido intervenidas; el resto de ellas (37.5\%) no regresaron a los controles. No se encontraron complicaciones derivadas de la intervención o de la medicación administrada al alta ni en el seguimiento.

Conclusiones: teniendo en cuenta las condiciones socioeconómicas de las pacientes con anomalías congénitas que se embarazan y el acceso al sistema de salud, el cierre percutáneo de defectos congénitos, en el posparto inmediato con dispositivo es seguro y eficaz, sin que se demuestren complicaciones inherentes al procedimiento o a la administración de antiplaquetarios. (Acta Med Colomb 2014; 39: 159-164).

Palabras clave: cardiopatía congénita, comunicación interauricular, ductus arterioso, embarazo, corazón, mortalidad materna.

\footnotetext{
Abstract

Introduction: congenital heart diseases in pregnancy are a major cause of maternal morbidity and mortality, with all the social and economic consequences that this implicates. So far corrections of heart diseases detected during pregnancy are performed in the subsequent six months after delivery, taking into account the reversal of the physiological changes proper to pregnancy, and its lack of treatment increases morbidity and mortality. In our environment deferred corrections are not performed because of the difficulties in the health system and the sociocultural conditions of patients, which imply loss of monitoring that impedes the timely correction of the defects and causing development of Eisenmenger syndrome.
}

Dr. Juan M. Senior S.: FACP. Cardiólogo Intervencionista, Jefe de Posgrado Cardiología Clínica e Intervencionista, Universidad de Antioquia, Hospital Universitario San Vicente de Paúl; Dr. Andrés Fernández C.: Cardiólogo Intervencionista Hospital Universitario San Vicente de Paúl; Dr. Arturo Rodríguez D.: Cardiólogo Intervencionista Hospital Universitario San Vicente de Paúl; Dra. Diana P. Cárdenas: Residente de Medicina de Urgencias Universidad de Antioquia; Dr. Erick J. Trespalacios: Especialista en Medicina Interna, Residente de Cardiología Clínica, Universidad de Antioquia; Dr. Roberto C. Trespalacios: MSc. Matemático, Máster en Estadística; Dr. Jorge M. Gómez J.: Ginecólogo Obstetra, Profesor Titular, Universidad de Antioquia, Hospital Universitario San Vicente Fundación. Medellín (Colombia).

Correspondencia. Dr. Juan Manuel Senior S. Medellín (Colombia).

E-mail: mmbt@une.net.co

Recibido: 07/VI/2013 Aceptado: 03/III/2014 
Objective: evaluate safety of percutaneous correction of congenital heart disease in women in their early postpartum days and the outcomes at short and long term.

Methods: descriptive study. Case series.

Results: eight cases of percutaneous closure with successful outcome are reported in four patients with atrial septal defect and four with patent ductus arteriosus in the first days after delivery (average of 8.7 days) with good clinical results for the newborn (average one minute APGAR score 6.8 and 8.7 at five minutes), and for the mother, with early hospital discharge (87.5\% in the first 24 hours after the procedure), with $62.5 \%$ of asymptomatic mothers at nine months after having been intervened; the rest of them $(37.5 \%)$ did not return to the controls. No complications from surgery or medication administered at discharge or follow-up were found.

Conclusions: considering the socio-economic conditions of the patients with congenital heart disease who become pregnant and their access to health care, percutaneous closure of congenital defects in the immediate postpartum with device is safe and effective. No complications inherent to the procedure or to the administration of antiplatelets were found. (Acta Med Colomb 2014; 39: 159-164).

Keywords: congenital heart disease, atrial septal defect, ductus arteriosus, pregnancy, heart, maternal mortality.

\section{Introducción}

Las cardiopatías son unas de las anomalías más frecuentes al nacimiento (1), afectando aproximadamente a $0.8 \%$ de todos los nacidos vivos (2). Las enfermedades cardiacas en el embarazo son causa importante de morbimortalidad materna (2) y las pacientes con cardiopatías congénitas representan un grupo especial, puesto que 5-10\% de ellas tendrán algún grado de hipertensión pulmonar que afectará su clase funcional y la sobrevida a largo plazo, con mortalidad de hasta $30-50 \%$ en quienes desarrollen síndrome de Eisenmenger $(9,10)$. Además se ha encontrado que la presencia de cardiopatías congénitas se asocia a partos pretérmino $(21 \%)$, hemorragia posparto $(14 \%)$, ruptura prematura de membranas $(10 \%)$, hipertensión asociada al embarazo, desprendimiento de placenta (3\%) y muerte fetal intrauterina (2\%). A largo plazo una de cada 10 mujeres en el posparto, presentan eventos cardiacos tardíos, de los cuales las arritmias y la falla cardiaca son los más comunes.

El servicio de cardiología del Hospital San Vicente de Paúl, ha tenido como práctica habitual el cierre de los cortocircuitos de izquierda a derecha detectados durante el embarazo, a los seis meses después del parto debido a que en ese momento se ha alcanzado nuevamente las condiciones hemodinámicas pregestacionales, se evita la posible interacción entre la lactancia y el uso de ácido acetil salicílico y/o clopidogrel y que el recién nacido tiene edad suficiente para permitirle a la madre someterse a la intervención; sin embargo, existe una alta tasa de pérdidas en el seguimiento. La ausencia de corrección de los defectos del septo interauricular podría desencadenar la presencia de síntomas, algunas veces más allá de la quinta década de la vida, como intolerancia al ejercicio, arritmias supraventriculares sintomáticas, embolismos paradójicos, falla cardiaca o falla cardiaca derecha y enfermedad vascular pulmonar (5-9\% de los pacientes). Una disminución de la distensibilidad ventricular de cualquier causa, desencadenará un cortocircuito de izquierda a derecha a través del defecto auricular, empeorando los síntomas (7). El objetivo de este trabajo es evaluar la seguridad de la corrección percutánea de cardiopatías congénitas tipo comunicación interauricular (CIA) y ductus arterioso persistente (DAP) en mujeres en los primeros días después de su parto y los resultados de la misma a corto y largo plazo.

\section{Material y métodos}

En el periodo comprendido entre abril de 2007 y febrero de 2011, se recibieron 16 partos de madres con cardiopatías congénitas tipo CIA o DAP, de las cuales $50 \%$ se dio de alta para corrección posterior ambulatoria que nunca se realizó por las dificultades en el sistema de salud y/o las condiciones idiosincráticas de las pacientes (Datos no publicados, Cifras del Servicio de Ginecología y Obstetricia, Hospital Universitario San Vicente de Paúl).

Estadística. Para este estudio, se utilizaron métodos estadísticos descriptivos. Se describen los datos y se muestran las medidas de tendencia central (media, mediana) y de dispersión (varianza y desviación estándar). Además, se realizó un diagrama de cajas para describir la distribución de los datos y mostrar la ubicación de la mediana respecto a los datos y con esto ver si los datos se encuentran sesgados o no. También se efectuó una prueba de hipótesis para comprobar si las medias de las medidas, en cada uno de los métodos diagnósticos, son estadísticamente iguales. Para esto, se utiliza el estadístico t-student con N-2 grados de libertad y un nivel de confianza de $95 \%$; además, se verifica también la prueba de hipótesis utilizando el valor de p correspondiente al valor del estadístico t-student encontrado. Se realizó un análisis descriptivo de los datos; para esto se midieron las correlaciones de las diferentes variables y se muestran los gráficos comparativos de algunas variables. Además, se contrastó el valor de p con el nivel de significancia $\alpha=(100-95 \%)$ para las pruebas de significancia del coeficiente de correlación. Para realizar los cálculos estadísticos y los gráficos, se utilizo el paquete estadístico 
minitab. Se recolectaron los datos de las pacientes en el momento del procedimiento, obteniendo a partir de la revisión de historias clínicas los datos mostrados en las Tablas 1 a 3 y se revisaron posteriormente las historias clínicas para extraer la información de los controles en consulta externa. La recolección de la información fue realizada por un autor utilizando una tabla de excel, y al finalizar, los datos fueron nuevamente revisados por un segundo autor para disminuir la posibilidad de error. Un tercer autor participó en la evaluación inicial, elección y cierre percutáneo de los defectos de todas las pacientes.

\section{Resultados}

En el periodo comprendido entre mayo de 2009 y mayo de 2011 se lograron realizar ocho cierres percutáneos de cortocircuitos de izquierda a derecha en pacientes que estaban en su postparto temprano, 4 DAP y 4 CIA tipo ostium secundum (OS); todas las pacientes pertenecientes al régimen subsidiado. El promedio de edad fue de 22 años (rango 18 a 28 años), el promedio de edad gestacional al momento del parto fue de 37.6 semanas (34.5 a 40.4 semanas), dos de las ocho pacientes tenían $<37$ semanas de gestación $(25 \%)$. Dos de las ocho pacientes tuvieron parto por vía vaginal, el resto fue por cesárea, por indicación obstétrica. Sólo un recién nacido tuvo complicaciones y requirió traslado a UCI neonatal, la razón para este pobre resultado obstétrico fue una circular de cordón que no fue posible resolver, generando un muy bajo APGAR al minuto 1 y 5 ; esta paciente tuvo parto vaginal. El APGAR promedio fue 6.8 al minuto 1 (rango 3 a 9) y 8.7 al minuto 5 (rango 6 a 10).

La clase funcional al ingreso al hospital de las pacientes era de acuerdo a la New York Heart Association (NYHA) I. El cierre percutáneo se hizo en promedio a los 8.7 días (rango 6 a 15 días) y el egreso se dio las 24 horas del cierre percutáneo para siete de las pacientes; una paciente estuvo hospitalizada seis días posterior al cierre del defecto, debido a un síndrome febril que se clasificó como una infección urinaria (hemocultivos negativos) y un mes después del regreso, en control con ginecología estaba asintomática (Tabla 1).

Todas las pacientes fueron evaluadas con ecocardiografía transtorácica, con la que se hizo el diagnóstico del defecto congénito y luego del parto fueron llevadas a hemodinamia para cierre percutáneo con dispositivo tipo amplatzer. En promedio las pacientes tenían un Qp/Qs (relación flujo pulmonar/flujo sistémico) de 2.1 (rango 1.6-3.1). Se documentó en todas las pacientes una buena función sistólica del ventrículo izquierdo, con FEVI 71\% (rango 60-80\%) y función diastólica normal en todas; una paciente tenía dilatación de la aurícula izquierda $\left(21 \mathrm{~cm}^{2}\right)$, y tres pacientes (37\%) tenían dilatación del ventrículo izquierdo, dado por un diámetro en fin de diástole $>5.4 \mathrm{~cm}$, con un promedio de $4.8 \mathrm{~cm}$ (rango 3.3 a $6.1 \mathrm{~cm}$ ). Se documentó dilatación del ventrículo derecho en tres pacientes (37\%) y de la aurícula derecha en cuatro pacientes (50\%) con un área promedio de $17.5 \mathrm{~cm}^{2}$ (rango 12-24 $\mathrm{cm}^{2}$ ); sin embargo, todas las pacientes tenían buena función sistólica del ventrículo derecho. El $87.5 \%$ de las pacientes tenía hipertensión arterial pulmonar, estimada por la velocidad de regurgitación tricuspídea, con un promedio de $40 \mathrm{mmHg}$ (rango $25-53 \mathrm{mmHg}$ ).

El tamaño del defecto en los pacientes con CIA tipo OS tuvo una media de $16.75 \mathrm{~mm}$ medidas por ecocardiografía, con una desviación estándar de 10.75; y por hemodinamia tuvo una media de $22.25 \mathrm{~mm}$, con una desviación estándar de 10.9.

Realizando una prueba de hipótesis, la inferencia que se hizo al asociar las medidas para el tamaño del defecto en los pacientes con CIA tipo OS tomadas por ecocardiografía $\mathrm{y}$ hemodinamia indica que no existe diferencia significativa

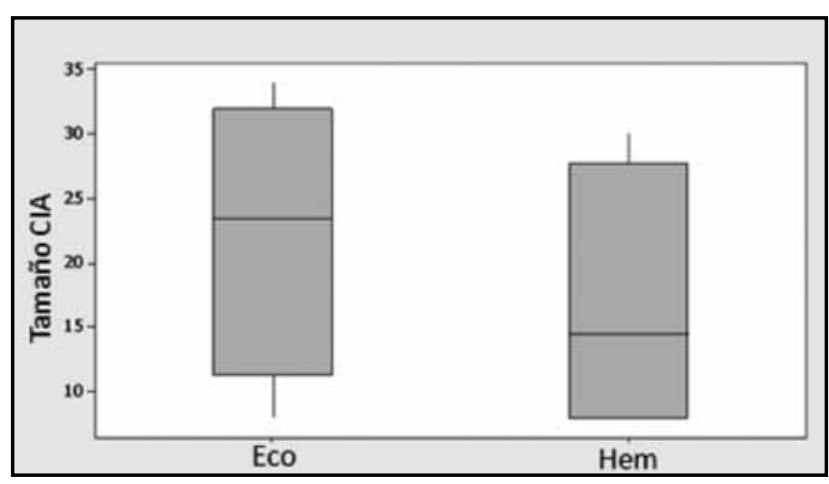

Figura 1. Correlación del tamaño de la CIA medida por ecocardiografía vs hemodinamia.

Tabla 1. Características de las pacientes, controles prenatales y seguimiento.

\begin{tabular}{|c|c|c|c|c|c|c|c|c|}
\hline Pte & Tipo & Edad & Qp/Qs & Tamaño por Hemodinamia & Tamaño por Ecocardiografía & Dimensión VD /AD & Dimensión VID-VIS/AI & PSAP \\
\hline 1 & CIA OS & 20 & 2.1 & $26 \mathrm{~mm}$ & $8 \mathrm{~mm}$ & $3.2 \mathrm{~cm} / 22 \mathrm{~cm}^{2}$ & $3.9-2.3 \mathrm{~cm} / 13 \mathrm{~cm}^{2}$ & 48 \\
\hline 2 & CIA OS & 18 & 3.1 & $21 \mathrm{~mm}$ & $21 \mathrm{~mm}$ & $3.1 \mathrm{~cm} / 24 \mathrm{~cm}^{2}$ & $4.0-2.7 \mathrm{~cm} / 18 \mathrm{~cm}^{2}$ & 42 \\
\hline 3 & CIA OS & 20 & 3 & $34 \mathrm{~mm}$ & $30 \mathrm{~mm}$ & $3.6 \mathrm{~cm} / 16 \mathrm{~cm}^{2}$ & $3.3-2.0 \mathrm{~cm} / 15 \mathrm{~cm}^{2}$ & 47 \\
\hline 4 & DAP & 19 & 1.9 & Aort $1.2 \mathrm{~mm}$, Pul $2.3 \mathrm{~mm}$ & & $1.4 \mathrm{~cm} / 12 \mathrm{~cm}^{2}$ & $4.8-2.8 \mathrm{~cm} / 11 \mathrm{~cm}^{2}$ & 53 \\
\hline 5 & DAP & 28 & 1.6 & Aort $10 \mathrm{~mm}$, Pulm $9 \mathrm{~mm}$ & aort $08 \mathrm{~mm}$, pulm $7 \mathrm{~mm}$ & $1.8 \mathrm{~cm} / 12 \mathrm{~cm}^{2}$ & $5.9-3.4 \mathrm{~cm} / 11 \mathrm{~cm}^{2}$ & $<30$ \\
\hline 6 & DAP & 28 & 1.7 & Aort $11 \mathrm{~mm}$, Pulm $5 \mathrm{~mm}$ & aor $2.2 \mathrm{~mm}$, pulm $2.8 \mathrm{~mm}$ & $1.6 \mathrm{~cm} / 14 \mathrm{~cm}^{2}$ & $6.1-3.9 \mathrm{~cm} / 18 \mathrm{~cm}^{2}$ & 35 \\
\hline 7 & CIA OS & 18 & 1.9 & $26 \mathrm{~mm}$ & $8 \mathrm{~mm}$ & $2.3 \mathrm{~cm} / 19 \mathrm{~cm}^{2}$ & $4.7-2.0 \mathrm{~cm} / 15 \mathrm{~cm}^{2}$ & 41 \\
\hline 8 & DAP & 25 & 2 & Aort $10 \mathrm{~mm}$, Pulm $10 \mathrm{~mm}$ & & $2.4 \mathrm{~cm} / 23 \mathrm{~cm}^{2}$ & $5.6-3.2 \mathrm{~cm} / 21 \mathrm{~cm}^{2}$ & 35 \\
\hline
\end{tabular}




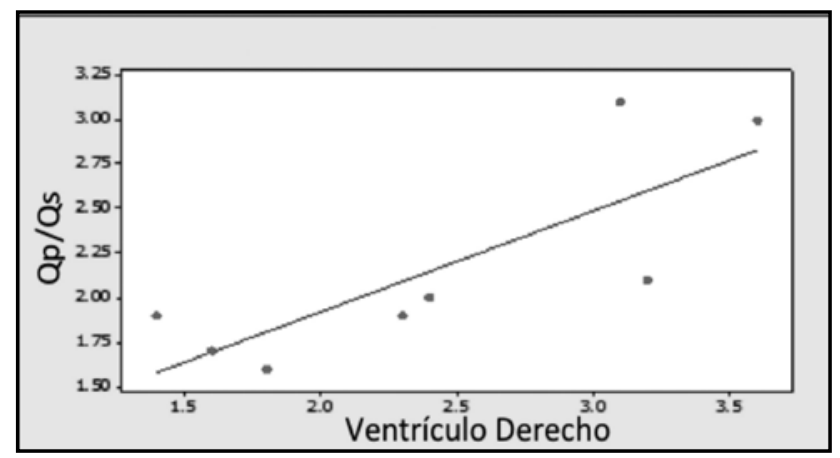

Figura 2. Correlación entre el tamaño del ventrículo derecho y el cortocircuito.

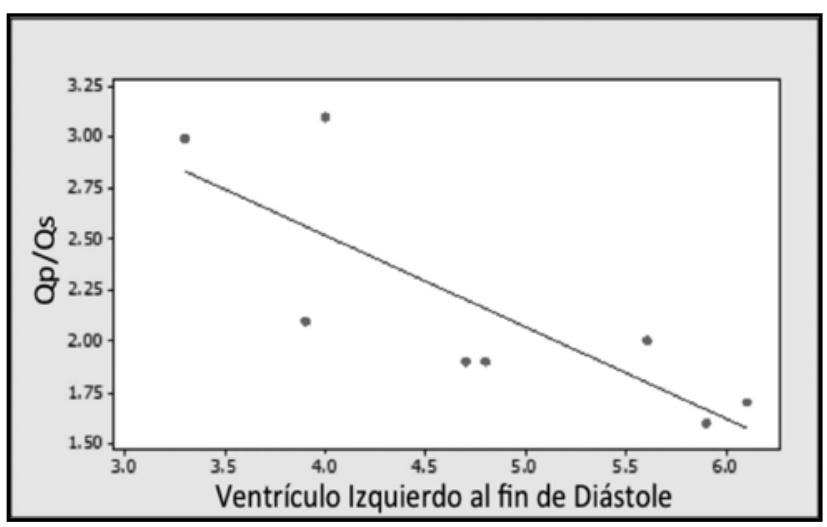

Figura 3. Correlación entre el tamaño del ventrículo izquierdo y el cortocircuito.

entre ellas ( $\mathrm{P}=>0.05$, IC $95 \%(-13.23,24.23))$ (Figura 1). La mediana de los datos para hemodinamia y ecocardiografía se encuentran detallados en la Figura 1.

El cierre de la CIA se hizo bajo visión ecocardiográfica y fluoroscópica de acuerdo con la técnica convencional y el cierre de DAP bajo visión fluoroscópica. En todos se utilizó el dispositivo tipo Amplatzer. El procedimiento fue exitoso en $100 \%$ de los casos, sin cortocircuito residual en la evaluación fluoroscopica ni en el control ecocardiográfico, inmediatamente posterior a la finalización del procedimiento. No se presentaron complicaciones durante el procedimiento.

La relación de $\mathrm{Qp} / \mathrm{Qs}$ y el tamaño del ventrículo derecho tuvieron una correlación de 0.808 y una relación inversa con el tamaño del ventrículo izquierdo al fin de la diástole; cuya correlación es -0.806 (Figuras 2 y 3). No se encontró una alta correlación entre el Qp/Qs y la aurícula derecha, la aurícula izquierda y la presión sistólica de la arteria pulmonar (PSAP). En cinco pacientes $(62.5 \%)$ se logró hacer un control posterior al procedimiento, todas ellas asintomáticas, y se logró realizar un seguimiento promedio de nueve meses en estas cinco pacientes ( 0.25 a 17 meses). No fue posible contactar a las otras tres pacientes, y tampoco regresaron para control por ginecoobstetricia, ni cardiología, lo que significa pérdida de $37.5 \%$ de las pacientes. A todas las pacientes se les recomendó tomar ácido acetilsalicílico
$100 \mathrm{mg}$ y clopidogrel $75 \mathrm{mg}$ orales diarios durante al menos tres meses (3-6 meses). En las Tabla 1 se muestran las características.

\section{Discusión}

En el reporte de la XXXII Conferencia de Bethesda en el año 2000 se estimó que existían aproximadamente 2800 adultos con cardiopatías congénitas por cada millón de personas, y más de la mitad de ellos con defectos moderados a severos (3). Las enfermedades cardiacas en el embarazo son causa importante de morbimortalidad materna y un grave problema de salud pública (2), pues una muerte materna es un indicador fidedigno del grado de desarrollo económico y social de un país, así como de las condiciones de equidad entre los géneros, del acceso a los servicios de salud, de las condiciones sociales y de oportunidades económicas para la mujer, y se traduce en niños que muy posiblemente abandonarán sus estudios y engrosarán las filas de trabajo infantil, de delincuencia juvenil y prostitución (4). El 5-10\% de las pacientes con cardiopatías congénitas desarrollan algún grado de hipertensión pulmonar, que afecta su clase funcional y la sobrevida y que en casos severos la presencia del síndrome de Eisenmenger tiene una mortalidad entre 30$50 \%$, y puede desencadenar abortos en $40 \%$ de los casos, $30 \%$ cursan con restricción del crecimiento intrauterino y la muerte materna puede ocurrir en el parto y hasta la primera semana posparto $(9,10)$. En un estudio prospectivo se encontró que una de cada diez mujeres en el posparto, presentan eventos cardiacos tardíos, de los cuales las arritmias y la falla cardiaca son los más comunes. Las mujeres en mayor riesgo de presentarlos son las que tienen una pobre clase funcional de base (NYHA III y IV) y/o cianosis, disfunción ventricular izquierda y obstrucción al tracto de salida del ventrículo izquierdo. Los eventos cardiacos maternos adversos fueron más frecuentes en mujeres que tuvieron eventos durante el embarazo (13).

El espectro de cardiopatías congénitas es amplio, desde defectos complejos que producen serias discapacidades e incluso la muerte en la infancia, hasta defectos menores que son descubiertos en adultos asintomáticos. Debido a los avances tecnológicos, estos defectos son ahora más frecuentemente detectados y corregidos, mejorando la sobrevida de las personas que los padecen. Por estas razones, muchas mujeres con cardiopatías congénitas alcanzan la edad reproductiva, lo que obliga a incluir componentes críticos de su cuidado como consejo preconcepcional y un manejo cuidadoso del embarazo y el parto $(2,5)$.

Los cambios fisiológicos del embarazo comienzan a partir de la sexta semana de gestación alcanzando su punto máximo a la semana 32, que incluyen aumento del volumen sanguíneo hasta en 50\% sobre el nivel pregestacional, aumento en la frecuencia cardiaca y del gasto cardiaco, disminución del retorno venoso y del gasto cardiaco en $25-30 \%$ asociado a la posición supina y disminución de la resistencia sistémica y pulmonar hasta el término del embarazo. Durante el 
trabajo de parto y parto, hay un aumento del gasto cardiaco hasta $50 \%$, vasoconstricción esplácnica, aumento de la presión venosa central, la precarga ventricular y el gasto cardiaco, y desde las primeras 48 horas posparto hay un aumento en la diuresis y regreso progresivo a los valores hemodinámicos pregestacionales entre la semana 4 y 12 , hasta el sexto mes $(2,6)$. Esta carga hemodinámica puede servir como una prueba fisiológica de estrés, y en mujeres con enfermedad cardiaca subyacente puede desenmascarar una reserva cardiaca limitada. El aumento en el estrés de la pared cardiaca puede impactar un corazón estructuralmente anormal, empeorando su funcionamiento y desencadenando procesos como apoptosis, fibrosis y arritmogénesis (13). Estos cambios pueden hacer manifiesta la presencia de defectos cardiacos congénitos, como la CIA y el DAP, como se reporta en la actual serie, permitiendo un diagnóstico y tratamiento oportunos.

Los defectos del tabique interauricular constituyen $10 \%$ de todos los defectos congénitos cardiacos, de los cuales $80 \%$ son tipo ostium secundum. Debido a las complicaciones a largo plazo, y que el riesgo de embolismo paradójico que se aumenta durante el embarazo y en el posparto (7), existen indicaciones claras respecto a la reparación de la comunicación interauricular $(7,14)$. El éxito del cierre percutáneo es mayor de $90 \%$, y tiene las ventajas de cursar con menos complicaciones y menor tiempo hospitalario.

El ductus arterioso persistente constituye $7 \%$ de todos los defectos cardiacos congénitos. La no corrección del defecto se asocia con algún riesgo de endarteritis, que puede aumentar con la edad y se asocia a dilatación de las cavidades izquierdas e hipertensión pulmonar relacionada con el cortocircuito (que puede ser revertido con la reparación del defecto) (7). La corrección percutánea tiene una mortalidad que se acerca a $0 \%$ (11), y las indicaciones también se han definido claramente $(7,12,14)$.

Reportamos el cierre exitoso de dos de las cardiopatías congénitas más frecuentes observadas en adultos, en el periodo del posparto inmediato, con baja tasa de complicaciones y $0 \%$ de mortalidad. En $62.5 \%$ de las pacientes se logró hacer un control posterior al procedimiento, obteniendo un seguimiento promedio de nueve meses en estas cinco pacientes (0.25-17 meses), sin que se hayan documentado síntomas de origen cardiovascular ni complicaciones secundarias al procedimiento en la madre que no ha completado su estado hemodinámico pregestacional o a la administración de antiagregantes plaquetarios que se iniciaron desde el posparto inmediato. La técnica de cierre no implica cambios sustanciales con respecto a los pacientes convencionales y las medidas obtenidas por ecocardiografía y angiografía se correlacionan, lo que permite definir en forma adecuada el tamaño del dispositivo a implantar y por lo tanto el éxito del procedimiento.

Ninguna de nuestras pacientes pertenece al régimen contributivo, lo que refleja sus bajas condiciones socioeconómicas, siendo una población vulnerable con dificultades de acceso a los servicios de salud, lo que pudo influir en la pérdida del seguimiento de $37.5 \%$ de las pacientes, y que sumado a $50 \%$ de las pacientes conocidas en los años previos que tenían estos defectos y que nunca regresaron a la consulta para iniciar el proceso de reparación, podría apoyar aún más la conducta de realizar cierres tempranos en la misma hospitalización del parto.

El Qp/Qs (relación flujo pulmonar/flujo sistémico) de nuestras pacientes era en promedio 2.1, lo que significa que los cortocircuitos tenían repercusión hemodinámica importante, y por lo tanto eran pacientes con altas posibilidades de desarrollar las complicaciones antes mencionadas, y en caso de no haberse corregido los defectos, tendrían una alta probabilidad de complicaciones maternofetales en embarazos posteriores o deterioro en su calidad de vida per $s e$, sin embargo hay que tener en cuenta que estas medidas pueden estar alteradas por las condiciones hemodinámicas inherentes a la gestación, ya mencionadas.

Las principales limitaciones de este estudio es el número de pacientes intervenidas, que refleja un tamaño de muestra pequeño, y la pérdida del seguimiento de varias de ellas por las condiciones inherentes a este grupo poblacional; sin embargo, el éxito de los resultados hace necesaria la realización de trabajos multicéntricos con una muestra mayor para verificar su reproductibilidad.

\section{Conclusiones}

Teniendo en cuenta las condiciones socioeconómicas de las pacientes con anomalías congénitas que se embarazan y el acceso al sistema de salud, el cierre percutáneo de defectos congénitos, como la comunicación interauricular tipo ostium secundum y el ductus arterioso persistente, en el posparto inmediato con dispositivo tipo Amplatzer es seguro y eficaz, sin que se demuestren complicaciones inherentes al procedimiento o a la administración de antiplaquetarios.

\section{Financiación}

Universidad de Antioquia, Grupo para el estudio de las enfermedades cardiovasculares.

\section{Conflictos de interés}

Ninguno.

\section{Referencias}

1. Congenital anomalies in Canada. A perinatal health report 2002. Minister of Public Works and Government Service Canada. http://publications.gc.ca/collections/ Collection/H39-641-2002E.pdf

2. Harris I. Management of pregnancy in patients with congenital heart disease Prog Cardiovasc Dis 2011; 53: 305-311.

3. Warnes CA, Liberthson R, Danielson GK et al. Task force 1: the changing profile of congenital heart disease in adult life. J Am Coll Cardiol 2001; 37: 1170- 1175.

4. Karam M, Bustamante P, Campuzano M. Aspectos sociales de la mortalidad Materna. Estudio de caso en el estado de Mexico. Medician Social 2007; 2: 205 211.

5. ACOG technical bulletin. Cardiac disease in pregnancy. Int J Gynaecol Obstet 1993; 41: 298-306.

6. Clark SL, Cotton DB, Lee $\mathbf{W}$ et al. Central hemodynamic assessment of norma term pregnancy. Am J Obstet Gynecol 1989; 161: 1439-1442.

7. Silversides C, Marelli A, Beauchesne L. Canadian Cardiovascualr Society 2009 Consensus Conference on the management of adults with congenital heart Disease. Can J Cardiol 2010; 26: 143-150. 
8. Fernandes S, Arendt K, Landzberg M. Pregnant women with congenital heart disease:cardiac, anesthetic and obstetrical implications. Expert Rev Cardiovasc Ther 2010; 8: 439-448.

9. Naguib M.A, Dob DP, Gatzoulis MA. Functional understanding of moderate to complex congenital heart disease and the impact of pregnancy. Int J Obstet Anesth 2010; 19: 306-312

10. Baumgartner $\mathbf{H}$, Bonhofer P, DeGroot $\mathbf{N}$ et al. ESC guidelines for the management of grown up congenital heart disease new version. Eur Heart J 2010; 31: 2915-2957.

11. Hirsh J, Devaney E, Ohye R, Bove E. The Heart: II Congenital Heart Disease.
En: Doherty GM, editor. Current Diagnosis \& Treatment Surgery. 13 ed. McGraw Hill 2010: paginas 392-423.

12. Warnes C. Pregnancy and Heart disease. En: Bonow R, Zipes D, Mann D, Libby P, eds. Braunwald Heart Disease. Textbook of cardiovascular medicine, 9 ed Elsevier Saunders Philadelphia 2012: paginas 1170-1780.

13. Balint O, Siu S, Mason J. Cardiac outcomes after pregnancy in women with Congenital Heart Disease. Heart 2010; 96: 1656-1661.

14. Warnes C, Williams R, Bashore T et al. ACC/AHA 2008 guidelines fo the management of adults with congenital heart disease. Circulation 2008; 118: 714-833. 